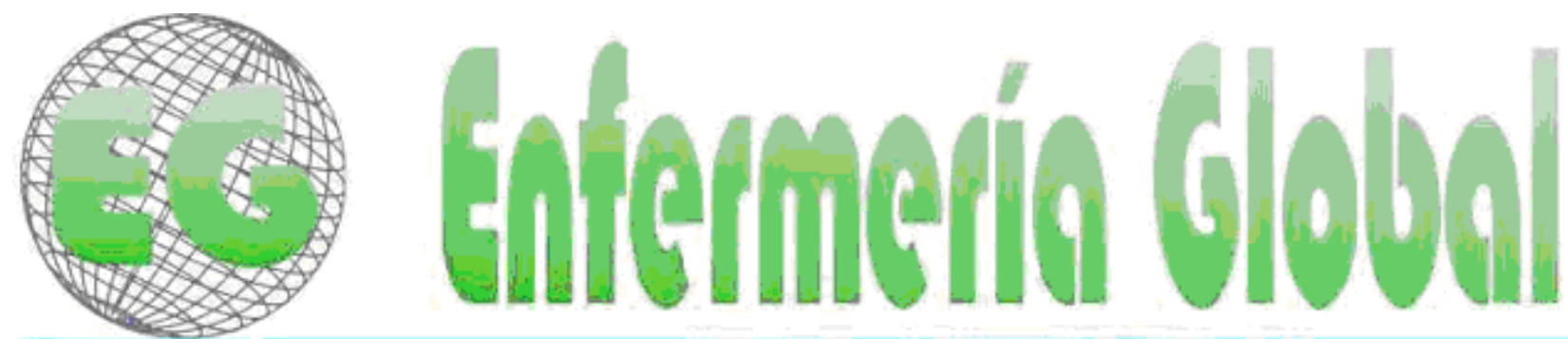

15SN 1696-6*5:

Revista electrónica cuatrimestral de Enfermeria

$\mathrm{N}^{\circ} 20$

Octubre 2010

www.um.es/egloball

\title{
CLÍNICA
}

\section{GESTACIÓN DE RIESGO: PERCEPCIÓN Y SENTIMIENTOS DE LAS MUJERES EMBARAZADAS CON AMNIORREXE PREMATURO}

GESTACÃO DE RISCO: PERCEPCÃO E SENTIMENTOS DAS GESTANTES COM AMNIORREXE PREMATURA

\section{*Costa, M da C., "**Bezerra Filho, JG., ${ }^{* * *}$ Andrade Bezerra, MG., "***Veríssimo} de Oliveira, MI., ${ }^{* * * * *}$ Carvalho de Oliveira, RM., ${ }^{* * * *}$ De Vasconcelos Silva, AR.

\author{
"Enfermera. UNIFOR, Ceará. "*Doctor em Salud Pública. Docente del Programa de Posgraduación \\ UFC/UECE. ***Máster en Enfermería, Profesora de la Universidad de Fortaleza, Enfermera de la Maternidad \\ Escuela Assis Chateaubriand-Universidad Federal de Ceará. **** Máster en Salud Pública, Enfermera de la \\ Maternidad Escuela Assis Chateaubriand-Universidad Federal de Ceará. ${ }^{* * * * *}$ Mestre en Enfermería, \\ Fortaleza, Ceará. Brasil. \\ Palabras clave: Embarazo de alto riesgo. Emociones. Conocimiento. Mujeres Embarazadas \\ Palavras-chave: Gravidez de Alto Risco. Emoções. Conhecimentos. Gestantes.
}

Keywords: High Risk Pregnancy, Emotions, Knowledge, and Pregnant Women

\section{RESUMEN}

Amniorrexe prematuro, embarazo de riesgo, se convirtió en preocupación mundial debido a los agravios en la gestante y en el feto. Objetivamos identificar el conocimiento de las gestantes sobre amniorrexe prematura y conocer sus percepciones y sus sentimientos ante esta patología. Estudio descriptivo con un enfoque cualitativo, desarrollado en una maternidad pública. Participaron 11 gestantes hospitalizadas con diagnóstico de amniorrexe prematura, en los meses de septiembre y octubre de 2007. Se utilizó en la recogida de datos entrevista semi-estructurada a partir de la cual surgieron las categorías: conocimiento de las gestantes en relación con amniorrexe prematura, que requieren la información, los sentimientos experimentados, la reacción ante la ruptura de la bolsa de aguas. Los resultados mostraron que las mujeres saben poco de la enfermedad, el temor por la vida de sus hijos y no saben qué hacer ante esta situación. De ahí la necesidad de los profesionales de la salud de mirar a las gestantes como seres que necesitan de apoyo y comprensión. 


\section{RESUMO}

Amniorrexe prematura, gestação de risco, tornou-se preocupação mundial devido os agravos na gestante e no feto. Objetivamos identificar o conhecimento de gestantes sobre amniorrexe prematura e conhecer suas percepções e seus sentimentos diante desta patologia. Estudo descritivo com abordagem qualitativa, desenvolvido em uma maternidade pública. Participaram 11 gestantes internadas com o diagnóstico de amniorrexe prematura, nos meses de setembro e outubro de 2007. Utilizou-se na coleta de dados entrevista semi-estruturada de onde emergiram as categorias: conhecimento das gestantes em relação à amniorrexe prematura, necessitando de informações, sentimentos vivenciados, reação diante do rompimento da bolsa das águas. Os resultados revelaram que as gestantes pouco conhecem da patologia, temem pela vida dos filhos e não sabem o que fazer diante dessa situação. Consideramos então a necessidade dos profissionais de saúde olhar para as gestantes como seres que necessitam de apoio e compreensão.

\section{ABSTRACT}

Premature amniorrhexis, risky pregnancy, became a global concern because of the harm to the mother and fetus. Aim to identify the knowledge of women of premature amniorrhexis and get to know their perceptions and their feelings about this pathology. Descriptive study with a qualitative approach, developed in a public maternity ward. 11 women participated while hospitalized with a diagnosis of premature amniorrhexis, in the months of September and October 2007. The collection of semistructured data was used, from which emerged the categories: Women's knowledge in relation to premature amniorrhexis, requiring information, and their feelings experienced, and reaction before their water broke. The results showed that women know little of the disease, or fear for the life of their children, and do not know what to do before that. Additionally health care professionals should regard women as human beings that need support and understanding.

\section{INTRODUCCIÓN}

El embarazo acarrea alteraciones emocionales y fisiológicas en las mujeres, pues ocurren cambios en el cuerpo, en el ámbito familiar y en las relaciones interpersonales. Así, la adaptación psicológica a las nuevas condiciones establecidas ante un embarazo requiere una reflexión de la mujer embarazada y de los profesionales de la salud frente a este ciclo vital.

En este sentido, debe saberse que algunos factores pueden desequilibrar la frágil estructura emocional de una mujer embarazada, impidiendo su proceso de adaptación a los desafíos impuestos por el embarazo. Si esto ocurre, podrá causar daños a su salud, desencadenando un embarazo de riesgo.

El embarazo de riesgo hace mucho que viene siendo discutido, tornándose una preocupación mundial, debido a los agravios que comprometen tanto a la mujer embarazada como al feto, que conduce al proceso de la enfermedad que resulta en tratamientos de alto costo, impactando en la economía del país.

Etimológicamente, la palabra riesgo se deriva de la palabra riscare, es decir, se atreven. Así, el riesgo sería una opción y no un destino. Para el Ministerio de Salud, la evaluación del riesgo no es tarea fácil, ya que el concepto de riesgo se asocia a varias posibilidades y la unión entre un factor de riesgo y un daño no siempre es explicada. Específicamente, el concepto de riesgo en el embarazo parece identificar los grados de vulnerabilidad durante los períodos de embarazo, parto, posparto y la vida del niño en su primer año. En la atención prenatal, el embarazo de alto riesgo se refiere a las alteraciones relacionadas tanto con la madre como con el feto ${ }^{1}$.

El embarazo entraña en sí mismo un riesgo para la madre y el feto. Sin embargo, en aproximadamente $20 \%$ de ellas, este riesgo se incrementa, siendo entonces incluidas entre los 
llamados embarazos de alto riesgo, en contraste a las "normales", llamadas de bajo riesgo. Así, se define como un embarazo de alto riesgo "aquel en que la vida o la salud de la madre y / o del feto tienen mayores posibilidades de ser atendidas que las de la media de la población considerada"2 $^{2}$

Para algunos autores ${ }^{3}$ entre los embarazos considerados de riesgo se encuentra la rotura prematura de membranas, o sea, la rotura de las membranas ovulares se produjo antes del inicio del parto, independientemente de la edad gestacional.

Varios autores ${ }^{4}$ explican que esta rotura prematura de membranas ovulares (RPM) es una condición común que afecta al 10\% de los embarazos, este hecho se produce cuando hay una solución de continuidad del corion y el amnios, ya que estas membranas limitan la cavidad amniótica durante todo el embarazo antes del inicio del trabajo del parto.

La incidencia de la rotura prematura de membranas (RPM) varía del $3 \%$ al $18,5 \%$. Alrededor del $8 \%$ al $10 \%$ de los pacientes con embarazo a término tienen esta patologia ${ }^{5}$. Cuando ocurre antes de la $37^{\mathrm{a}}$ semanas es clasificado como rotura prematura de membranas, que tiene una incidencia de variación del 3\% al 19\% de todos los embarazos y es responsable en cerca del $30 \%$ de todos los partos prematuros ${ }^{3}$.

En la visión de los autores ${ }^{6}$ cuando el embarazo se encuentra a término, el parto ocurrirá espontáneamente dentro de 24 horas en $80 \%$ de los casos. Cuanto más lejos del término, mayor será el periodo de latencia. Esto período corresponde al intervalo entre la rotura de las membranas y el parto. Cuanto mayor sea este intervalo, mayores serán los riesgos de infecciones maternas, fetales o neonatales. La rotura se prolonga cuando este período de latencia dura más de 24 horas, y cuanto menor sea la edad gestacional mayor será este periodo.

En cuanto a los indicadores de riesgo para la rotura prematura de membranas se pueden considerar: las infecciones maternas del tracto urinario, cervicitis (micoplasma, clamidilla y urea plasma) y las enfermedades de transmisión sexual (gonorrea, clamidia y tricomonas), las infecciones intrauterinas; incompetencia istmocervical; gestación múltiple; polihidramnios; tabaquismo; deficiencia nutricional; la historia previa de la rotura de membranas y antecedentes familiares de la rotura de membranas.

Por consiguiente, la rotura prematura de membranas es un fenómeno complejo y de riesgo obstétrico, porque su etiología es poco conocida, su diagnóstico es difícil, los riesgos para las madres y para los bebés son considerables y su manejo es controvertido.

En esta perspectiva, ante el número considerado de mujeres embarazadas con rotura de membranas es necesario examinar si las mujeres al desarrollar un embarazo de riesgo requieren un tratamiento y cuidados especiales, sobre todo en los aspectos emocionales. Por lo tanto, es importante evaluar la comprensión de estas mujeres acerca de la rotura de membranas y del impacto de estas informaciones con el fin de minimizar los factores de estrés, ya que la mujer embarazada en la que se identificó un factor de riesgo, podrá intensificar sus dificultades para adaptaciones emocionales. Conforme afirman algunos autores ${ }^{2}$, el mero hecho de recibir la identificación de "alto riesgo" es suficiente para que la mujer embarazada se identifique como diferente en contraposición con embarazo de bajo riesgo (normal).

Entonces, teniendo en cuenta las necesidades de las mujeres embarazadas, las lagunas existentes en enfermería acerca de estudios sobre aminorrexe prematura, la importancia de desarrollar este conocimiento, combinado con nuestro interés sobre el tema, y fundamentados 
en autores ${ }^{7}$ sobre la definición de que percibir está conectado a la adquisición de conocimientos a través de los sentidos, con énfasis en la visión, se optó por desarrollar este estudio que tiene como objetivo identificar el conocimiento de estas mujeres embarazadas acerca de la rotura de membranas y conocer sus percepciones y sus sentimientos ante de un embarazo de alto riesgo.

El conocimiento, tan profundo como posible, de las adaptaciones del organismo materno es el paso indispensable. Quien no conoce la fisiología del embarazo no sabrá valorar debidamente las reclamaciones $\mathrm{y}$, como consecuencia, hará diagnóstico incorrecto y establecerá el tratamiento ineficaz o iatrogênico ${ }^{2}$.

\section{METODOLOGÍA}

Se trata de un estudio descriptivo con abordaje cualitativo, que contiene entre sus preceptos cualitativos la descripción, en que el investigador observa, cuenta, describe y clasifica.

El estudio fue realizado en una maternidad pública, en la ciudad de Fortaleza, Ceará-Brasil, clasificada por el Ministerio de Salud, como de nivel terciario, considerado como un centro de referencia para todo el estado del Ceará. Tiene como misión promover la formación de recursos humanos, actividades de aprendizaje, educación, investigación y extensión, en la búsqueda de la excelencia en la atención general y la salud humanizada de las mujeres y los recién nacidos. Esta maternidad tiene en su estructura física un espacio con 44 camas destinadas para la admisión de mujeres embarazadas en situación de riesgo y un lugar con sillas, mesas, sonido y televisión en que las mujeres embarazadas desarrollan artesanías y conferencias.

Participaron del estudio 11 mujeres embarazadas con diagnóstico de rotura de membranas sin importar color, raza, religión o nivel de educación, internadas en los meses de septiembre y octubre de 2007 en la maternidad que albergó el estudio. La cantidad de participantes se hizo a partir de la saturación de los datos.

Se utilizó para la colecta de datos una entrevista semi-estructurada dividida en dos partes. La primera formada por los datos de identificación y obstetricia. La segunda guiada por tres cuestiones norteadores, a saber: ¿Qué sabe usted sobre amniorrexe prematura? ¿Cómo es para usted la vivencia de este momento? ¿Cuénteme usted cómo reaccionó cuando vio o supo que su bolsa de aguas se rompió?

Como un recurso para grabar las informaciones se utilizó una grabadora para la validación de la entrevista, después de la entrevista los investigadores hicieron un retroceso en las citas con la finalidad de que la entrevistada escuchase la entrevista, confirmase o no de acuerdo con lo que fue registrado; caso de queestuviese en desacuerdo, la participante sería nuevamente entrevistada hasta la confirmación. Las entrevistas fueron posteriormente transcritas en la íntegra.

Después de la transcripción de los testimonios y lectura detallada de las respuestas, los datos obtenidos en estas lecturas fueron organizados de acuerdo con la convergencia de los discursos, en la cual surgieron las categorías empíricas que fueran fundamentadas en la literatura relacionada con la temática. De acuerdo con algunos autores ${ }^{8}$ esta es una manera de organizar los datos mediante su agrupamiento en categorías. Cuando terminó este paso fueron seleccionados los testimonios considerados significativos, con el fin de autenticar las conclusiones y sus significados. 
El estudio fue presentado al Comité de Ética e Investigación. En cuanto a los aspectos éticos se siguieron las directrices y las normas de regulación contenidas en la Resolución 196/96 relativa a la investigación con seres humanos obedeciendo los cuatro principios básicos de la bioética que son: la autonomía, no maleficencia, beneficencia y justicia.

Las participantes firmaron un formulario de consentimiento libre y esclarecido, formulario este que tiene dos copias, siendo una copia del sujeto de la investigación y otra del investigador, en el cual contenía los objetivos de la investigación, y fue informado acerca de la divulgación de los datos proporcionados.

\section{ANÁLISIS, ORGANIZACIÓN Y PRESENTACIÓN DE LOS RESULTADOS.}

Participaron del estudio 11 mujeres embarazadas que recibieron código de los sentimientos o virtudes a fin de mantener el anonimato de la información. Para facilitar la comprensión del lector organizamos los resultados en dos fases, la primera llamada de caracterización de las participantes, en que las mujeres se presentaron en un marco y el segundo fue la descripción de las categorías.

Quadro 1: Caracterización de los participantes del estudio (MEAC-UFC, Fortaleza, Ceará, Brasil, 2007).

\begin{tabular}{|c|c|c|c|c|c|c|c|}
\hline Codinomes & $\begin{array}{l}\text { Añ } \\
\text { os }\end{array}$ & $\begin{array}{l}\text { Grado de } \\
\text { instrucci } \\
\text { ón }\end{array}$ & $\begin{array}{l}\text { Estado } \\
\text { civil }\end{array}$ & $\begin{array}{l}\text { Pré- } \\
\text { natal }\end{array}$ & \begin{tabular}{|l|} 
Edads \\
Gestacio \\
nal \\
(semanas \\
\end{tabular} & $\begin{array}{l}\text { No de } \\
\text { gestaci } \\
\text { ones }\end{array}$ & $\begin{array}{l}\text { Número } \\
\text { de abortos }\end{array}$ \\
\hline Amor & 20 & $\begin{array}{l}2^{0} \text { grado } \\
\text { completo }\end{array}$ & soltera & 6 & 32 & 1 & 0 \\
\hline $\mathrm{Paz}$ & 20 & $\begin{array}{l}1^{\circ} \text { grado } \\
\text { completo }\end{array}$ & soltera & 3 & 30 & 2 & 0 \\
\hline Felicidad & 20 & $\begin{array}{l}2^{\circ} \text { grado } \\
\text { completo }\end{array}$ & \begin{tabular}{|l} 
unión \\
consens \\
ual
\end{tabular} & 6 & 31 & 1 & 0 \\
\hline Esperanza & 34 & $\begin{array}{l}1^{\circ} \text { grado } \\
\text { incomplet } \\
0^{2}\end{array}$ & soltera & 6 & 26 & 9 & 0 \\
\hline Caridad & 25 & $\begin{array}{l}\text { Superior } \\
\text { incomplet } \\
\text { o }\end{array}$ & soltera & 2 & 25 & 1 & 0 \\
\hline Coraje & 27 & $\begin{array}{l}1^{\circ} \text { grado } \\
\text { incomplet } \\
0^{2}\end{array}$ & soltera & 2 & 33 & 6 & 5 \\
\hline $\begin{array}{l}\text { Perseveran } \\
\text { cia }\end{array}$ & 38 & $\begin{array}{l}\text { Superior } \\
\text { incomplet } \\
\text { o }\end{array}$ & casada & 6 & 31 & 1 & 0 \\
\hline Fé & 21 & $\begin{array}{l}1^{\circ} \text { grado } \\
\text { incomplet } \\
0^{-}\end{array}$ & \begin{tabular}{|l} 
unión \\
consens \\
ual
\end{tabular} & $\begin{array}{l}\text { no } \\
\text { recuer } \\
\text { da }\end{array}$ & 30 & 4 & 0 \\
\hline Cariño & 22 & $\begin{array}{l}1^{\circ} \text { grado } \\
\text { incomplet } \\
0\end{array}$ & $\begin{array}{l}\text { unión } \\
\text { consens } \\
\text { ual }\end{array}$ & $\begin{array}{l}\text { No } \\
\text { recuer } \\
\text { da }\end{array}$ & 33 & 3 & 0 \\
\hline
\end{tabular}




\begin{tabular}{|l|l|l|l|l|l|l|l|}
\hline Amistad & 41 & $\begin{array}{l}2^{0} \text { grado } \\
\text { incomplet } \\
0\end{array}$ & $\begin{array}{l}\text { unión } \\
\text { consens } \\
\text { ual }\end{array}$ & 3 & 31 & 3 & 1 \\
\hline Humildad & 32 & $\begin{array}{l}1^{\circ} \text { grado } \\
\text { completo }\end{array}$ & casada & 1 & 28 & 6 & 0 \\
\hline
\end{tabular}

Fonte: MEAC-UFC

Según los resultados, los participantes se encuentran en el grupo etáreo entre 20 y 41 años, llamamos la atención para las mujeres embarazadas con edad superior a 35 años consideradas por el Ministerio de Salud ${ }^{9}$ edad de riesgo para el embarazo.

El grado de instrucción predominante fue la escuela primaria incompleta, con cuatro mujeres, hay también dos con educación primaria terminada, dos con educación secundaria completa, una con educación secundaria incompleta y dos estaban en la educación superior.

Sabemos que la educación influye en todos los aspectos, dejando claro que si una persona no tiene una educación adecuada, probablemente no tendrá buenas condiciones financieras, o que daría lugar a una mala salud. El conocimiento de la salud que los individuos por sí solos no pueden lograr, se define por factores como la pobreza y el desempleo, el analfabetismo y subvivienda, la falta de servicios básicos de saneamiento, el agua y los factores sociales ${ }^{10}$.

En relación al tipo de unión encontramos cinco solteras, dos se casaron y cuatro en una unión consensual. Con respecto a la edad gestacional, cuatro estaban respectivamente con 25, 26, 28 semanas y dos con 30 semanas, dos con 33 semanas, y tres con 31 semanas.

En cuanto al número de embarazos, tres estaban al mismo tiempo en el segundo, cuarto y noveno embarazo, cuatro en el primer embarazo, dos en el tercer embarazo y dos en el sexto embarazo. Siendo que entre estas, una tuvo un aborto involuntario y otra tuvo cinco.

En cuanto a la consulta de prenatal, cuatro mujeres embarazadas hicieron seis consultaciones, dos no sabían, dos hicieron dos consultaciones, dos hicieron tres consultaciones y una hizo una consultación.

Sabemos que la atención prenatal es un factor importante en la reducción de la mortalidad perinatal, lo que se comprende fácilmente, ya que muchas enfermedades maternas que afectan al feto pueden ser tratadas o controladas en este periodo, evitando efectos perjudiciales para el producto. También hay que recordar que un prenatal adecuado ciertamente guiará en el sentido de evitar algunos problemas específicos del parto o incluso para algunos cuidados de atención inmediata de los recién nacidos como la incompatibilidad sanguínea ${ }^{11}$.

El control de la atención prenatal, de acuerdo con las recomendaciones oficiales de la salud debe comenzar temprano, tener la cobertura universal, ser realizado periódicamente, estar integrado con otras acciones preventivas y curativas, y debe ser observado un número mínimo de visitas. Su éxito depende, en gran parte, del momento de su comienzo y el número de consultas realizadas. Este número varía según el mes de inicio y las complicaciones durante el embarazo. El Ministerio de Salud recomienda al menos seis visitas prenatales para un embarazo a término en mujeres embarazadas sin factores de riesgo identificados, con inicio temprano, hasta el cuarto mes de embarazo. El intervalo entre las consultas no debe exceder de ocho semanas ${ }^{12}$.

Podemos entonces ver que de las 11 mujeres embarazadas, sólo cuatro hicieron las visitas recomendadas para una atención prenatal efectiva, lo que nos lleva a reflexionar sobre la 
posibilidad de que el riesgo de amniorrexe hubiera sido detectado previamente y que estas mujeres podrían estar preparadas para un embarazo con atención. Cuestionamos acerca de la calidad de esta atención prenatal, ya que los factores de riesgo se pueden detectar y se pueden tomar medidas para minimizar un embarazo de alto riesgo.

\section{Categorías}

De acuerdo con los testimonios de las mujeres fueron extraídas cuatro categorías temáticas, a saber, el conocimiento de las mujeres embarazadas en relación con la rotura prematura de membranas, lo que requiere información, sentimientos vivenciados, reacciones delante de la rotura de la bolsa de aguas, que serán descritas a continuación.

\section{Conocimiento de las mujeres embarazadas en relación con la rotura prematura de membranas}

En esta categoría, las declaraciones revelaron que ocho de las participantes ignoran la patología y tres relacionaron su etiología a otros factores distintos de la rotura de membranas, como embarazo agitado, edad avanzada y el esfuerzo físico. Lo que nos hace pensar que estamos ante un fracaso en la comunicación entre el profesional de salud y las mujeres embarazadas durante el tratamiento, los profesionales de alguna manera pueden estar explicando, sin embargo, las mujeres embarazadas no están entendiendo lo que observamos en los discursos de las mujeres:

"... Lo que sé es que realmente son pocas cosas, incluso sé muy pocas cosas sobre eso. "(Perseverancia).

"Sólo dije que era para yo quedar, y quedar en la estancia hospitalaria." (Coraje).

"... y ellos dicen que la pérdida de líquido se da realmente por un factor que ellos aún no saben cómo decir lo que es exactamente..."(Fe).

El proceso de comunicación no sólo depende de la escucha, sino de una serie de factores como, con quién se está hablando, bajo qué condiciones el acto está ocurriendo (número de los intercolocutores y ruido) y el objetivo de la comunicación (social, de trabajo y de negocios) ${ }^{13}$.

En este sentido, las informaciones deberían ser realizadas en forma de diálogo, ya que la finalidad del diálogo es sobrepasar la atención individual, es explorar cuestiones vistas como complejas o difíciles, sobre varios puntos de vista, y su resultado es una libre exploración que trae a cabo la profundidad de la experiencia y del pensamiento de las personas, que puede ir más adelante de sus puntos de vista individuales. A través del diálogo se puede observar el propio pensamiento y, luego percibirlo como activo, siendo por tanto posible asumir una postura de participación con la creatividad para resolver los problemas del cotidiano. Por lo tanto, creemos que el diálogo puede permitir que los individuos expresen sus necesidades, sus deseos y sus pensamientos y prepararse/negociar la forma como satisfacerlas ${ }^{14}$.

\section{Exigir el diálogo / información}

Sabemos que la mujer necesita de información sobre su salud y su derecho a desarrollar un embarazo saludable. En esta categoría encontramos que las mujeres embarazadas necesitan de información, como se muestra en los testimonios a seguir: 
"... No sé, creo que... yo tuve un horror de un hijo y nunca tuve esto problema. ¿Será porque el niño es pequeño? "(Esperanza).

"Es así, ellos no dan la explicación totalmente como es, ellos sólo observan más la persona (...) me dieron dos inyecciones para fortalecer los pulmones del niño cuando llegué aquí. Y pasé siete días tomando ampicilina y ahora estoy sin suero, sin nada, simplemente tome el hierro y estoy en observación, sólo eso mismo. "(Paz).

La asistencia durante la gestación tiene como objetivo dar la bienvenida a la mujer desde el comienzo del embarazo, asegurando su terminación, el nacimiento de un niño sano, y asegurar el bienestar de la madre y su recién nacido. Para que esta atención ocurra el Ministerio recomienda la incorporación de conductas acogedoras ${ }^{15}$.

En esta perspectiva, los profesionales que trabajan con las mujeres embarazadas deben verlas con una "concepción de la persona humana", tratando de establecer mecanismos de interacción que desvelen las verdaderas necesidades y sus significados. No deben asumir una posición superior, viendo a las mujeres embarazadas como personas indefensas, débiles y sumisas. Si el servicio y los profesionales asumen una posición de igualdad, respeto y confianza en relación con su experiencias y conocimientos adquiridos, la relación será de un desarrollo emocional y de crecimiento mutuo, teniendo en cuenta las necesidades biopsicosociales y culturales de la mujer embarazada ${ }^{16}$.

\section{Sentimientos de las mujeres}

Muchos sentimientos fueran anotados, entre ellos la tristeza, la angustia, el miedo, la preocupación, la ansiedad, la calma y la voluntad de huir.

Los sentimientos, el miedo y la preocupación fueron evidentes, por el hecho de la mujer no saber lo que podría haber ocurrido o lo que está aconteciendo con sus hijos dentro del vientre ahora sin la bolsa íntegra para protegerlos. Para las personas legas, el hecho de la rotura de la bolsa significa que su hijo nacerá poco después, lo que crea preocupación y miedo cuando ello no ocurre. Esto nos alerta sobre la importancia de disponer atención integral a las mujeres embarazadas, proveer las aclaraciones necesarias para tranquilizarlas sobre la salud de su bebé. Las entrevistadas así se expresaron:

"... es una cosa que usted queda aguardando, no sabe el momento, cuándo y cómo va a ser, si va a ser cierto o no. Estaba preocupada. "(Caridad).

"Tuve miedo, yo tuve miedo de morir aquella hora. Yo y el bebé "(Humildad).

"Para mi yo iba a perder..." (Coraje).

En las lecturas de los discursos, podemos inferir que la tristeza expresa es dada por el hecho de que ninguna mujer quiere, ni está dispuesta, para que en lugar de tener un embarazo tranquilo, tenga que quedar internada con la posibilidad de perder a su hijo, como fue manifestado en las siguientes declaraciones:

"Para mí está siendo triste ¿eh? De estar aquí dentro, una cosa que nunca sucedió comigo, ¿eh?"(Paz). 
"Yo tuve la expectativa de quedar embarazada y todo, quedé embarazada, me gustaría mucho mantener la (...) tristeza por la situación en que yo me encuentro ¿eh?" (Perseverancia).

"(...) Hace ocho días mañana que estoy acá, ellos no deciden una cosa ni otra.

"(Humildad).

La posibilidad de un parto prematuro se rompe con el sueño de tener el niño en el tiempo normal, libre de complicaciones una vez que las condiciones físicas causadas por enfermedades maternas o relacionadas con el embarazo afectan en el proceso del embarazo y pueden resultar en hospitalización ${ }^{17}$.

El tiempo prolongado de hospitalización nos lleva a la conclusión de que este fue el motivo de que las participantes expresaran el sentimiento de angustia al manifestarar el deseo de regresar a sus hogares, por el desconocimiento de lo que está sucediendo con su familia. Aquí están los testimonios:

"Estoy muy angustiada, esperando alguna noticia si voy tener luego, o no voy. Sólo angustia, siento voluntad de volver para mí casa. "(Amor)

"Mi deseo es salir de aquí y volver para mí casa" (Cariño)

"Yo ya pedí a ella para volver a mí casa y ella me dijo que yo no podía no (...) acá estoy yo para quedar aquí hasta el día de mi parto". "Que no es para yo volver a mi casa" (Coraje)

Es necesario recordar que las mujeres durante muchos años fueron poseedoras de las prácticas de salud, cuyos significados persisten hoy y remiten la importancia de las mujeres en el proceso de cuidar de la família ${ }^{18}$. Observamos la fuerza de esta práctica cuando las mujeres expresaron su preocupación tanto por el niño que esperaban, como también con los niños que estaban en casa. Las participantes manifestaron:

"... Me preocupo más, es así, yo quedo pensando sólo en mis hijos que están en casa... "(Fe)

"... Creo que es difícil, siento dolor de cólico, por mi yo estaba en casa porque yo tengo una chica más joven, ella tiene 10 meses. "(Humildad).

"Yo creo que es malo quedar sin mi hijo que está al nacer pero no está comigo el otro. El hijo de mi hermana. Yo estoy, tengo pena de él porque es directo conmigo, yo me compadezco de él. Mi pensamiento está sólo en él aquí dentro.... "(Coraje).

\section{Reacción en el momento de romper la bolsa de aguas.}

En esta categoría observamos muchas actitudes diferentes, la gran mayoría quedó confusa al no saber la mujer si lo que estaba ocurriendo era una respuesta fisiológica o no, traducidas en los siguientes discursos:

"Lloré mucho pensando que mi hija iba a morir, sólo hice lo que yo supe hacer en este momento fue llorar." (Amor).

"Yo pensé que iba tener el bebé antes de tiempo, entonces nos ponemos nerviosos por el miedo de que algo suceda con el bebé." (Amistad). 
Pero algunas se mantuvieron tranquilas, en el momento cuando se dio cuenta que su bolsa se había roto, como se refiere en los discursos:

"Yo me mantuve muy tranquila hasta, que me vine con mucha tranquilidad y todo porque yo sabía que cuanto más nerviosa yo podría causar daño y perder, la pérdida sería aún mayor." (Perseverancia).

"Yo no estaba preocupada." (Fe).

Hay que tener en cuenta que las personas no siempre conocen las razones que les llevan a actuar de determinada manera en la vida cotidiana. La adecuada actuación del profesiona de la salud que busca la atención de las demandas reales por el cuidado de los usuarios de salud, es un gran desafío, cuyo sentido debe ser objeto de la dedicación de las instituciones de salud y de sus profesionales, especialmente los que trabajan en instituciones que atienden a la población de baja renta ${ }^{18}$.

\section{CONSIDERACIONES FINALES}

El estudio reveló que las mujeres embarazadas conocen poco la patología, tienen miedo por la vida de sus hijos y no saben lo que hacer ante esta situación. Por lo tanto el embarazo no debe ser un evento estresante en el sentido patológico, puesto que ya es fisiológicamente, y que los profesionales de la salud, especialmente el enfermero, debe estar atento a los cambios y alteraciones que puedan surgir durante un embarazo. El seguimiento del embarazo es importante, porque su objetivo no es dejar que ocurran trastornos para la madre, ya que afectará directamente al feto, que es sensible a factores emocionales. Cuando una mujer se enfrenta a un embarazo de alto riesgo podrá tener dificultades para responder de forma positiva al cambio de su cuerpo.

Ante estos dos factores el conocimiento y la percepción, pudieramos alertar a los profesionales de la salud en cuanto al cuidado de una mujer en esta etapa de la vida que en sí misma sólo trae una serie de sentimientos ambiguos, que hacen que el embarazo sea un momento único e intenso en cuanto a los sentimientos, especialmente el miedo y la preocupación. En caso de rotura prematura de membranas, el hecho de la gravedad es mucho más intensa, como la madre que no tiene conocimientos sobre su salud, no podrá participar activamente del proceso de gestación, desde el momento en que se dice solo que la bolsa de aguas rompió antes de la hora, que el bebé está bien y que tiene que esperar por el curso "normal" del embarazo, la madre crea una ansiedad en cuanto a lo que realmente está esperando.

Por lo tanto, considerando que la enfermería debe actuar identificando las inquietudes de las mujeres embarazadas con rotura de membrana, reduciendo el sufrimiento, aclarándolas acerca de la enfermedad, las formas del tratamiento, orientándolas para el auto-cuidado y prevención de infecciones, preparándolas para el enfrentamiento de posibles complicaciones en el feto y su internación en hospital. Por lo tanto, creemos que este cuidado y la contribución de las políticas públicas promuevan el acceso a los servicios de salud permitiendo la mejora de la calidad de la atención, con acciones que integran todos los niveles de atención: promoción, prevención y atención de la salud para las mujeres embarazadas y recién nacidos. 


\section{REFERÊNCIAS}

1. Gomes R, Cavalcanti LF, Marinho ASN, Silva LGP. Os Sentidos do Risco na Gravidez Segundo a Obstetrícia: um estudo bibliográfico. Revista Latino-Americana de Enfermagem. 2001; 9 (4): 62-67.

2. Tedesco JJA. A grávida: suas indagações e as duvidas do obstetra. São Paulo (SP): editora Atheneu; 2002.

3. Guedes RAA, Valença YMP, Oliveira RN, Lima, SRG. Prognóstico materno e perinatal em gestações complicadas por amniorrexe prematura. Ginecologia e Obstetrícia Atual 2001 jan./fev; 1 (2): 16-18.

4. Pierre AMMA, Bastos GZG, Oquendo RJ, Alencar CA. Repercussões maternas e perinatais da ruptura prematura das membranas até a $26^{a}$ semana gestacional. Revista Brasileira de Ginecologia e Obstetrícia 2003 mar; 25 (2): 109-114.

5. Assis JB, Martins DP. Amniorrexe prematura. Disponível em: http://www.paulomargotto.com.br/documentos/Amniorrexe\%20prematura.ppt. Acesso em: 26/03/07

6. Santos LC, Amorim MMR. Amniorrexe prematura - diagnostico e conduta. Revista Femina $2002 \mathrm{jan} / \mathrm{fev} ; 30$ (1): 21-28.

7. Ferreira $\mathrm{ABH}$. Dicionário Aurélio Básico da Língua Portuguesa. Rio de Janeiro: Nova Fronteira, 1988.

8. Lakatos EM, Marconi MA. Fundamentos de metodologia cientifica. $4^{\text {a }}$ ed. São Paulo (SP): Atlas; 2001.

9. Brasil. Ministério da Saúde (BR). Gestação de alto risco. Brasília (DF); 2000.

10. Santos ZMSA, Albuquerque VLM, Sampaio FHS. Vacinação - o que o usuário sabe? Revista Brasileira em Promoção da Saúde. 2005; 18 (1).

11. Laurenti R, Buchalla CM. Estudo da morbidade e da mortalidade perinatal em maternidades II - Mortalidade perinatal segundo peso ao nascer, idade materna, assistência pré-natal e hábito de fumar da mãe. Revista de Saúde Pública. [periódico on line] 2007 nov; [citado junho de 1985]; 19 (3): [aproximadamente 7 telas]:Disponível: http://www.scielo.br

12. Bettiol H, Aragão VMF, Ribeiro VS, Alves MTSSB, Mochel EG, Silva AAM, Coimbra LC. Fatores associados à inadequação do uso da assistência pré-natal. Revista Saúde Pública. [periódico on line] 2007 nov; [citado ago 2003]; 37 (4): [aproximadamente 7 telas]:Disponível: http://www.scielo.br

13. Batista ACM, Sampaio FM. Nível de satisfação dos idosos usuários de próteses auditivas doadas pela APC-NAMI-UNIFOR. Revista Brasileira em Promoção da Saúde. 2005; 18 (1): 7 10.

14. Cecagno D, Cecagno S, Siqueira $\mathrm{HCH}$. Trabalhador de enfermagem: agente colaborador no cumprimento da missão institucional. Revista Brasileira de Enfermagem. 2005 jan/fev; 58 (1): 22-26.

15. Cabral FB, Ressel LB, Landergahl MC. Consulta de enfermagem: estratégia de abordagem a gestante na perspectiva de gênero. Escola Ana Nery Revista de Enfermagem 2005 dez; 9 ( 3): 459-65. 
16. Falcone VM, Mäder CV, Nascimento CFL, Santos JMM, Nóbrega FJ. Atuação multiprofissional e a saúde mental de gestantes. Revista de Saúde Pública. 2005 ago; 39 (4): 612-618.

17. Silveira IP, Campos ACS, Carvalho FAM, Barroso MGT. Ação educativa a gestante fundamentada na promoção da saúde: uma reflexão. Escola Ana Nery Revista de Enfermagem $2005 \mathrm{dez} ; 9$ (3): 451-458.

18. Hoga LAK. Práticas de cuidado e cura desenvolvidas por mulheres no contexto doméstico. Revista Paulista de Enfermagem 2006 jul/set; (24): 140-147. 